\title{
Modelling the effect of fermentation temperature and time on starter culture growth, acidification and firmness in made-in-transit yoghurt
}

\begin{abstract}
Made-in-transit (MIT) is a new concept that aims to develop products that can undergo part of their manufacture during transportation to their market destination. Fermented foods are suited to developing into MIT products under carefully controlled conditions that need to be set through experimentation and modelling. In the present study, primary and secondary models for growth of Streptococcus thermophilus STM5 and Lactobacillus acidophilus LA5, starter culture used in MIT yoghurt were developed. Kinetic data obtained at a fermentation temperature of $22.5-30{ }^{\circ} \mathrm{C}$ for $168 \mathrm{~h}$ of fermentation time were used to fit into a primary model to determine the maximum specific growth rate ( $\mu$ max) and lag time $(\lambda)$. Among three primary models used to model the temperature effect on starter culture growth, the modified Gompertz model described the experimental data better than modified logistic and Baranyi models. The derived growth kinetic parameters, such as maximum specific growth rate ( $\mu$ max) and lag phase duration $(\lambda)$, were modelled using the square root equation as the secondary model. The selection of the best models was based on R2 (the coefficient of multiple determinations). The validation of the developed models was carried out by fermenting set yoghurt within (interpolation) and outside (extrapolation) the fermentation temperatures used to develop the models. The possibility for the prediction of starter bacteria growth will be useful for the quality assurance of made-in-transit products.
\end{abstract}

Keyword: Made-in-transit (MIT); Predictive microbiology; Fermentation 